\title{
An Efficient Convertible Undeniable Signature Scheme with Delegatable Verification
}

\author{
Jacob C. N. Schuldt and Kanta Matsuura \\ Institute of Industrial Science, University of Tokyo, \\ 4-6-1 Komaba, Meguro-ku, Tokyo 153-8505, Japan. \\ \{schuldt, kanta\}@iis.u-tokyo.ac.jp
}

\begin{abstract}
Undeniable signatures, introduced by Chaum and van Antwerpen, require a verifier to interact with the signer to verify a signature, and hence allow the signer to control the verifiability of his signatures. Convertible undeniable signatures, introduced by Boyar, Chaum, Damgård, and Pedersen, furthermore allow the signer to convert signatures to publicly verifiable ones by publicizing a verification token, either for individual signatures or for all signatures universally. In addition, the signer is able to delegate the ability to prove validity and convert signatures to a semi-trusted third party by providing a verification key. While the latter functionality is implemented by the early convertible undeniable signature schemes, most recent schemes do not consider this despite its practical appeal.

In this paper we present an updated definition and security model for schemes allowing delegation, and highlight a new essential security property, token soundness, which is not formally treated in the previous security models for convertible undeniable signatures. We then propose a new convertible undeniable signature scheme. The scheme allows delegation of verification and is provably secure in the standard model assuming the computational co-Diffie-Hellman problem, a closely related problem, and the decisional linear problem are hard. Our scheme is, to the best of our knowledge, the currently most efficient convertible undeniable signature scheme which provably fulfills all security requirements in the standard model.
\end{abstract}

Keywords: undeniable signatures, universal/selective convertibility, provable security

\section{Introduction}

Undeniable signatures, first introduced by Chaum and van Antwerpen [10], are like ordinary signatures, except that verification of a signature requires interaction with the signer. Unlike ordinary signatures, this enables a signer to control who can verify his signatures and when verification is allowed. This feature makes undeniable signatures attractive when sensitive data or confidential business agreements are being signed, since the signer is guaranteed that only the relevant parties can verify his signature and no outsider will be able to link him to the signed data. To preserve non-repudiation, an undeniable signature scheme furthermore requires that a signer is able to disavow an invalid signature. Hence, a signer will either be able to confirm or disavow the validity of any signature, and any dispute can be resolved by letting the signer convince a judge about the validity or invalidity of the signature in question. Since their introduction, a number of undeniable signature schemes have been proposed e.g. $[9,17,31,26,24,32,23]$.

In [6], Boyar, Chaum, Damgård and Pedersen introduced convertible undeniable signatures which allow the signer to convert his undeniable signatures into publicly verifiable signatures. Two types of conversions were introduced: selective conversion which enables the signer to individually convert signatures, and universal conversion which enables the signer to convert all (existing and future) signatures. A signer selectively converts a signature $\sigma$ on a message $m$ by releasing a token $t k_{\sigma}$ which will convince any verifier that $\sigma$ is indeed a valid signature 
on $m$. Likewise, a signer universally converts all signatures by releasing a universal token $t k_{*}$ which can be used as a token for any signature. This feature is desirable when public verifiability is required after a period of time, which, for example, is the case for the problem of keeping digital records of confidential political decision (e.g. see [15]). Another aspect of the definition in [6] is that the private key material of the signer is divided into two parts: a signing key $s k$ and a verification key $v k$. The former is only used to sign messages, whereas the latter is used to convert and confirm or disavow signatures. This property is useful in scenarios where the signer is met with more verification requests than he has capacity to handle, or the signer might become off-line or otherwise unavailable and therefor cannot handle verification requests. In such scenarios, the signer will be able to delegate the verification by releasing $v k$ to a semi-trusted entity who will then have the capacity to verify signatures on behalf of the signer. It is required that the scheme remains unforgeable, even for the semi-trusted entity with the knowledge of $v k$.

Overview of prior work. The original scheme by Boyar et al. [6] was shown to be insecure by Michels, Petersen and Horster [28] when an universal token is released. Michels et al. furthermore proposed an updated scheme, but only heuristic arguments for the security of this scheme were presented. Recently, Aimani and Vergnaud [4] provided an analysis of the updated scheme in the generic group model. Furthermore, Damgård and Pedersen [15] proposed two convertible undeniable signature schemes based on El Gamal signatures, but did not give full proofs of invisibility, and Michels and Stadler [29] proposed a scheme based on Schnorr signatures.

The first RSA based scheme was proposed by Gennaro, Rabin and Krawczyk [17] which Miyazaki later improved [30] (see also [18]). Kurosawa and Takagi [25] proposed a (selective convertible only) scheme which they claimed to be the first RSA based scheme secure in the standard model, but it was shown by Phong, Kurosawa and Ogata [34] that the scheme does not provide full invisibility. Phong et al. furthermore proposed a new selective and universally convertible RSA based scheme secure in the standard model.

Laguillaumie and Vergnaud [27] defined and proposed a pairing-based time-selective convertible undeniable signatures which allow conversion of signatures constructed in a given time period, and Monnerat and Vaudenay [31] pointed out that their MOVA undeniable signature scheme supports selective conversion although a formal analysis is not given. Recently, Huang, $\mathrm{Mu}$, Susilo and $\mathrm{Wu}[22]$ proposed the currently most efficient scheme in the random oracle model which supports both selective and universal conversion. Yuen, Au, Liu and Susilo [39] proposed a selective and universal convertible standard model scheme, but it was shown by Phong, Kurosawa and Ogata [33] that the scheme is not invisible for the standard definition of invisibility. Phong et al. furthermore proposed two efficient schemes which are claimed to be the first practical discrete logarithm based schemes both providing selective and universal conversion and being provably secure in the standard model. However, as we discuss in Section 4, these schemes do not provide token soundness. Lastly, Huang and Wong [20] proposed a scheme with even shorter signatures than the schemes by Phong et al., but only prove the scheme to be invisible according to a weaker definition of invisibility which does not guarantee signer anonymity. In fact, it is fairly easy to see that the scheme does not provide anonymity, which will be highlighted in Section 4.

An intuitive approach to the construction of a convertible undeniable signature scheme is to use an encryption scheme to encrypt (parts of) an ordinary signature, and this is indeed the approach used in $[15,33]$. Aimani $[2,3]$ proposed a generic construction based on a certain 
class of encryption and signature schemes. However, this approach does not provide selective conversion as described above; while a signer is able to extract a valid public verifiable signature from an undeniable signature, a verifier will not receive any proof that the received publicly verifiable signature corresponds to the undeniable signature i.e. the verifier does not receive a token which allows him to independently verify the undeniable signature, but only a publicly verifiable signature derived from the undeniable signature. It should be noted that a designated confirmer signature, in which the signer holds both signer and confirmer key pairs, will not automatically yield a selective and universal convertible signature scheme for a similar reason; the ability of the confirmer to extract a publicly verifiable signature from an undeniable signature does not necessarily imply the ability to provide a token which will convince a verifier of the validity of the original signature.

All of the early proposed schemes $[6,28,15,17]$ implement the above described separation of the signer's key material into a signer key and verification key, which allows delegation of the verification. However, despite the practical advantages of this property, it is not considered in the formalization, security model or the concrete schemes presented in most recent papers $[27,25,22,39,2,34,33]$ (we note that [20] discuss delegation of verification as an extension). In these schemes, only the ability of the signer to confirm, disavow and convert signatures is considered and no explicit mechanism is provided for delegating this ability. Note that although the possession of an universal token allows verification of any signature, this does not necessarily provide the ability to efficiently prove validity to a third party in a nontransferable way.

Our Contribution. We present an updated definition and security model for schemes allowing delegation, and highlight a new security property, token soundness, which is not formally treated in previous security models ${ }^{1}$. Token soundness guarantee that a malicious signer (or delegated verifier) cannot produce a token such that an invalid signature/message pair appears valid. This is different from the ordinary completeness requirement which only considers honestly generated tokens, and will furthermore, in combination with unforgeability of undeniable signatures, guarantee unforgeability of message/signature/token tuples (see Section 4). We also note that the recently proposed schemes by Phong, Kurosawa and Ogata [33] do not provide token soundness, and hence allows a malicious signer to fool potential verifiers.

We then propose a convertible undeniable signature scheme which allows verification delegation and is provably secure in the standard model assuming the computational co-DiffieHellman problem, a closely related problem, and the decisional linear problem are hard (see Section 2). Our scheme is the most efficient scheme, in terms of signature size, that provably fulfills all security properties of a convertible undeniable signature scheme in the standard model (see Section 6 for a comparison).

\section{Preliminaries}

Negligible function. A function $\epsilon: \mathbb{N} \rightarrow[0,1]$ is said to be negligible if for all $c>0$ there exists an $n_{c}$ such that for all $n>n_{c} e(n)<1 / n^{c}$.

Bilinear maps. Our scheme makes use of groups equipped with a bilinear map (we refer the reader to [5] for a detailed description of these maps). To instantiate our schemes, we consider

\footnotetext{
${ }^{1}$ We note that $[20]$ independently proposed a similar security property which they refer to as non-claimability, but that our results were made public [37] well ahead of those from [20].
} 
a generator $\mathcal{G}$ that on input $1^{k}$ outputs a description of groups $\mathbb{G}_{1}, \mathbb{G}_{2}$ and $\mathbb{G}_{T}$ of prime order $p$ where $2^{k}<p<2^{k+1}$, a bilinear map $e: \mathbb{G}_{1} \times \mathbb{G}_{2} \rightarrow \mathbb{G}_{T}$ and an isomorphism $\psi: \mathbb{G}_{2} \rightarrow \mathbb{G}_{1}$. We will use the notation $\mathbb{P}=\left(e, \mathbb{G}_{1}, \mathbb{G}_{2}, \mathbb{G}_{T}, p, \psi\right)$ as a shorthand for the output of $\mathcal{G}$.

The discrete logarithm problem. Given a group $\mathbb{G}$ of order $p$, where $2^{k}<p<2^{k+1}$, and random elements $g, h \in \mathbb{G}$, the discrete logarithm problem in $\mathbb{G}$ is defined as computing $x \in \mathbb{Z}_{p}$ such that $g_{2}^{x}=h$. We say that the discrete logarithm problem is hard in $\mathbb{G}_{2}$ if all polynomial time algorithms have negligible probability (in the parameter $k$ ) of solving the problem.

The computational co-Diffie-Hellman problem. Given $\mathbb{P}=\left(e, \mathbb{G}_{1}, \mathbb{G}_{2}, \mathbb{G}_{T}, p, \psi\right)$, elements $g_{2}, g_{2}^{a} \in$ $\mathbb{G}_{2}$ and $h \in \mathbb{G}_{1}$ where $a$ is a random element in $\mathbb{Z}_{p}$, the computational co-Diffie-Hellman problem in the bilinear groups $\left(\mathbb{G}_{1}, \mathbb{G}_{2}\right)$ is to compute $h^{a} \in \mathbb{G}_{1}$. We say the computational co-Diffie-Hellman problem is hard in $\left(\mathbb{G}_{1}, \mathbb{G}_{2}\right)$ if all polynomial time algorithms have negligible probability (in the parameter $k$ ) of solving the problem.

Besides the above defined problem, we will also consider the following variant: Given $\mathbb{P}=\left(e, \mathbb{G}_{1}, \mathbb{G}_{2}, \mathbb{G}_{T}, p, \psi\right)$, elements $g_{2}, g_{2}^{a}, h \in \mathbb{G}_{2}$ where $a$ is a random element in $\mathbb{Z}_{p}$, compute $\psi\left(h^{a}\right) \in \mathbb{G}_{1}$. We will refer to this problem as the computational $\psi$-Diffie-Hellman problem to distinguish it from the above, and say that the problem is hard in $\left(\mathbb{G}_{1}, \mathbb{G}_{2}\right)$ if all polynomial time algorithms have negligible probability (in the parameter $k$ ) of solving the problem.

The decisional linear problem. Given a group $\mathbb{G}$ of order $p$, where $2^{k}<p<2^{k+1}$, and elements $u, v, u^{x}, v^{y}, h, h^{z} \in \mathbb{G}$ where $x, y$ are random elements in $\mathbb{Z}_{p}$, the decisional linear problem in $\mathbb{G}$ is to decide whether $z=x+y$ or $z$ is a random element in $\mathbb{Z}_{p}$. We say the decisional linear problem is hard in $\mathbb{G}$ if all polynomial time algorithms have negligible probability (in the parameter $k$ ) of solving the the problem.

\section{Convertible Undeniable Signatures}

A convertible undeniable signature (CUS) scheme consists of the following algorithms and protocols:

- Setup: Given input $1^{k}$, this algorithm outputs a set of public parameters par.

- KeyGen: Given par, this algorithm outputs a public key $p k$, a private verification key $v k$ and a private signing key $s k$. The verification key $v k$ will be used as private prover input in the confirm/disavow protocols, and to selectively convert signatures, whereas the signing key will only be used to sign messages.

- Sign: Given par, sk and a message $m$, this algorithm outputs an undeniable signature $\sigma$.

- Convert: Given par, $v k$ and $(m, \sigma)$, this algorithm returns a verification token $t k_{\sigma}$ for $\sigma$ if $(m, \sigma)$ is a valid message/signature pair. Otherwise, the algorithm returns $\perp$.

- Verify: Given par, $p k,(m, \sigma)$ and $t k_{\sigma}$, this algorithm returns either accept or reject.

- (Confirm, $\left.\mathrm{V}_{c o n}\right)$ : A pair of interactive algorithms for confirming validity of a signature. Both algorithms take as common input par, $p k$ and $(m, \sigma)$. The algorithm Confirm takes as an additional private input the verification key $v k$. While Confirm has no local output, the algorithm $\mathrm{V}_{\text {con }}$ will output either accept or reject after having interacted with Confirm.

- (Disavow, $\mathrm{V}_{d i s}$ ): A pair of interactive algorithms for disavowing validity of a signature. Similar to the above, both algorithms take as common input par, $p k$ and $(m, \sigma)$, and the algorithm Disavow takes the verification key $v k$ as an additional private input. Disavow has no local output, but $\mathrm{V}_{\text {dis }}$ will output either accept or reject after having interacted with Disavow. 

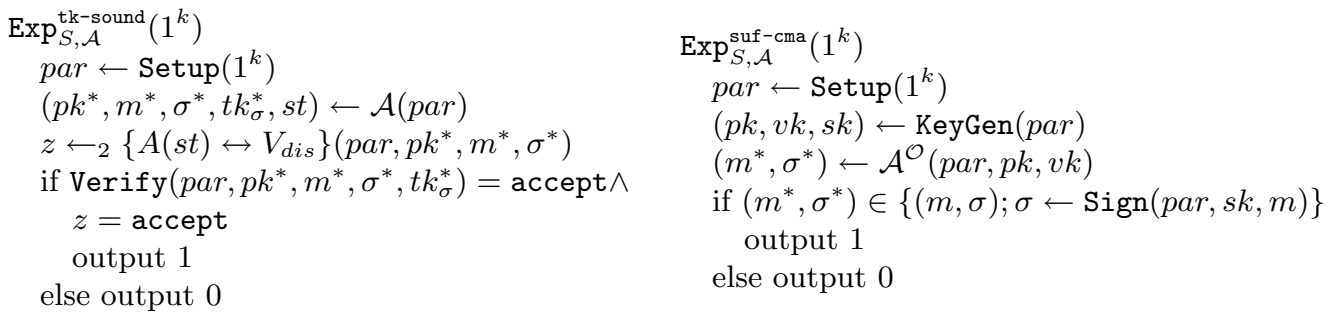

Fig. 1. Token soundness and unforgeability experiments

Note that the above definition does not explicitly mention how a universal token is generated or how signatures are verified using this token since this functionality follows directly from the separation of the private key material into a signing key $s k$ and a verification key $v k$. More precisely, a universal token corresponds to $v k$, and using this, any signature can be verified using the Convert algorithm. We assume that given $(p k, v k)$ it can be verified that $v k$ is a verification key for the public key $p k$.

We use the notation $\left\{\operatorname{Confirm}(v k) \leftrightarrow \mathrm{V}_{c o n}\right\}($ par, $p k, m, \sigma)$ to denote the interaction between Confirm and $\mathrm{V}_{c o n}$ with the common input $(p a r, p k, m, \sigma)$ and the private input $v k$ to the Confirm algorithm (a similar notation is used for Disavow and $\mathrm{V}_{\text {dis }}$ ). We furthermore use $z \leftarrow 2$ \{ Confirm $\left.(v k) \leftrightarrow \mathrm{V}_{c o n}\right\}(p a r, p k, m, \sigma)$ to denote the output of $V_{c o n}$ upon completion of the protocol i.e. $z$ will be either accept or reject.

Correctness. It is required that for all parameters par $\leftarrow \operatorname{Setup}\left(1^{k}\right)$, all keys $(p k, v k, s k) \leftarrow$ $\operatorname{KeyGen}($ par $)$, all messages $m \in\{0,1\}^{*}$, and all signatures $\sigma \leftarrow \operatorname{Sign}(p a r, s k, m)$, that the interaction $z \leftarrow_{2}\left\{\operatorname{Confirm}(v k) \leftrightarrow \mathrm{V}_{\text {con }}\right\}($ par, $p k, m, \sigma)$ yields $z=$ accept. Furthermore, it is required that for all $t k_{\sigma} \leftarrow \operatorname{Convert}(p a r, v k, m, \sigma)$ that Verify $\left(p a r, p k, m, \sigma, t k_{\sigma}\right)=$ accept. Lastly, it is required that for any $\left(m^{\prime}, \sigma^{\prime}\right) \notin\{(m, \sigma): \sigma \leftarrow \operatorname{Sign}(p a r, s k, m)\}$, the interaction $z^{\prime} \leftarrow_{2}\left\{\right.$ Disavow $\left.(v k) \leftrightarrow \mathrm{V}_{d i s}\right\}\left(\right.$ par $\left.\left., p k, m^{\prime}, \sigma^{\prime}\right)\right\}$ yields $z^{\prime}=$ accept.

\section{Security Model}

A CUS scheme is required to provide token soundness, unforgeability, invisibility and nonimpersonation, which will be defined below. Besides this, both the confirm and disavow protocols are required to be zero-knowledge proofs [19] to guarantee soundness of the protocols and transcript simulatability. The latter property will in combination with invisibility of the scheme provide what is commonly referred to as non-transferability (see below).

\subsection{Token Soundness}

The soundness of the confirm and disavow protocols intuitively captures the requirement that a signer cannot "cheat" when interacting with a verifier in the confirm or disavow protocol i.e. he cannot convince a verifier that a signature is both valid and invalid. However, it does not guarantee that a cheating signer cannot produce a token $t k_{\sigma}$ and a message/signature pair $(m, \sigma)$ such that Verify $\left(p a r, p k, m, \sigma, t k_{\sigma}\right)=$ accept, but $(m, \sigma)$ can be disavowed. This requirement, which we will refer to as token soundness, is somewhat surprisingly not formally treated in the used security models for convertible undeniable signatures. Token soundness of 
a CUS scheme $S$ is defined via the experiment $\operatorname{Exp}_{S, \mathcal{A}}^{\mathrm{tk}-\text { sound }}\left(1^{k}\right)$ shown in Figure 1. We define the advantage of the algorithm $\mathcal{A}$ as

$$
\operatorname{Adv}_{S, \mathcal{A}}^{\mathrm{tk}-\text { sound }}=\operatorname{Pr}\left[\operatorname{Exp}_{S, \mathcal{A}}^{\mathrm{tk}-\text { sound }}\left(1^{k}\right)=1\right]
$$

Definition 1 A CUS scheme is said to have token soundness if for all polynomial time algorithms $\mathcal{A}$, the advantage $\operatorname{Adv}_{S, \mathcal{A}}^{t k \text {-sound }}$ is negligible in the security parameter $k$.

We note that the recently proposed schemes by Phong, Kurosawa and Ogata [33] do not provide token soundness as defined above. More specifically, the schemes in [33] make use of NIZK proofs as tokens, and a signer will construct a token for a selective conversion of a signature by generating a NIZK proof of the validity of the signature. However, since the common reference string (CRS) used by the NIZK proofs is stored as part of the public signer key (i.e. the CRS will be generated by the signer), a malicious signer will, by the properties of the NIZK proofs, be able to generate a CRS which is indistinguishable from an honestly generated one, and which allows the signer to simulate the NIZK proofs. Hence, the malicious signer can break the token soundness of the schemes by simulating a NIZK proof for an invalid signature. This scenario can be avoid be letting the verifier generate the CRS, but this will tie a conversion to a single verifier and will not provide public verifiability. Alternatively, the CRS could be generate by a trusted third party. However, both of these options limit the practical applicability of the scheme.

\subsection{Unforgeability}

Strong unforgeability against a chosen message attack for a CUS scheme $S$ is defined via the experiment $\operatorname{Exp}_{S, \mathcal{A}}^{\text {suf-cma }}\left(1^{k}\right)$ shown in Figure 1. In the experiment, $\mathcal{A}$ has access to the oracle $\mathcal{O}=\left\{\mathcal{O}_{\text {Sign }}\right\}$ which is defined as follows

- $\mathcal{O}_{\text {Sign }}$ : Given a message $m$, the oracle returns $\sigma \leftarrow \operatorname{Sign}(p a r, s k, m)$.

It is required that $\mathcal{A} \operatorname{did}$ not obtain $\sigma^{*}$ by submitting $m^{*}$ to $\mathcal{O}_{\text {Sign }}$. Note that since $\mathcal{A}$ is given the verification key $v k, \mathcal{A}$ can convert signatures and run the confirm and disavow protocols by himself, and there is no need to provide $\mathcal{A}$ with oracles for these tasks. The advantage of $\mathcal{A}$ is defined as

$$
\operatorname{Adv}_{S, \mathcal{A}}^{\text {suf-cma }}=\operatorname{Pr}\left[\operatorname{Exp}_{S, \mathcal{A}}^{\text {suf-cma }}\left(1^{k}\right)=1\right]
$$

Definition 2 A CUS scheme is said to be strongly unforgeable if for all polynomial time algorithms $\mathcal{A}$, the advantage $\operatorname{Adv}_{S, \mathcal{A}}^{\text {suf-cma }}$ is negligible in the security parameter $k$.

While the above definition does not involve tokens, it will, in combination with token soundness, guarantee that an adversary without the knowledge of $s k$ cannot produce $\left(m, \sigma, t k_{\sigma}\right)$ such that $\operatorname{Verify}(p a r, p k, m, \sigma)=$ accept without having obtained $(m, \sigma)$ from the signer. This follows easily from the following observation. If the adversary does produce $\left(m, \sigma, t k_{\sigma}\right)$, then if $(m, \sigma)$ is a valid message/signature pair, the adversary has broken the above defined unforgeability property, whereas if $(m, \sigma)$ is not a valid message/signature pair, the token soundness of the scheme has been broken, which should not be possible even for an adversary knowing $s k$.

We furthermore stress the importance of giving $\mathcal{A}$ access to $v k$ in the above definition. This guarantees that if a signer delegates the verification operation by releasing $v k$ to a semitrusted entity, this entity will not be able to forge new signatures, but only verify existing ones. 


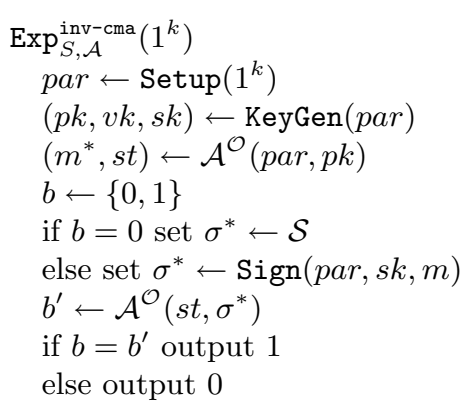

Fig. 2. Invisibility and non-impersonation experiments

\subsection{Invisibility}

Invisibility against a chosen message attack for a CUS scheme $S$ is defined via the experiment $\operatorname{Exp}_{S, \mathcal{A}}^{\mathrm{inv} \text {-cma }}\left(1^{k}\right)$ shown in Figure 2. In the experiment, $\mathcal{S}$ denotes the signature space and is defined as $\mathcal{S}=\{\sigma:(p k, v k, s k) \leftarrow \operatorname{KeyGen}(p a r) ; m \leftarrow \mathcal{M} ; \sigma \leftarrow \operatorname{Sign}(p a r, s k, m)\}$ where $\mathcal{M}$ is the message space given in par. Furthermore, $\mathcal{A}$ has access to the oracles $\mathcal{O}=\left\{\mathcal{O}_{\text {Sign }}, \mathcal{O}_{\text {Conv }}, \mathcal{O}_{\text {Conf } / \text { Dis }}\right\}$ which are defined as follows:

- $\mathcal{O}_{\text {Sign }}$ : Defined as in the unforgeability experiment.

- $\mathcal{O}_{\text {Conv }}$ : Given a message/signature pair $(m, \sigma)$, this oracle returns $t k_{\sigma} \leftarrow$ Convert $(p a r, v k, m, \sigma)$ if $(m, \sigma) \in\left\{\left(m^{\prime}, \sigma^{\prime}\right): \sigma^{\prime} \leftarrow \operatorname{Sign}(p a r, s k, m)\right\}$. Otherwise the oracle returns $\perp$.

- $\mathcal{O}_{\text {Conf/Dis }}$ : Given a message signature pair $(m, \sigma)$, this oracle interacts with $\mathcal{A}$ by running $\operatorname{Conf} \operatorname{irm}($ par, $v k, m, \sigma)$ if $(m, \sigma) \in\left\{\left(m^{\prime}, \sigma^{\prime}\right): \sigma^{\prime} \leftarrow \operatorname{Sign}(\right.$ par, sk, $\left.m)\right\}$. Otherwise, the oracle interacts with $\mathcal{A}$ by running $\operatorname{Disavow}($ par, $v k, m, \sigma)$.

It is required that $\mathcal{A}$ does not query $\left(m^{*}, \sigma^{*}\right)$ to the convert or confirm/disavow oracles. We define the advantage of $\mathcal{A}$ in the experiment as

$$
\operatorname{Adv}_{S, \mathcal{A}}^{\text {inv-cma }}=\left|\operatorname{Pr}\left[\operatorname{Exp}_{S, \mathcal{A}}^{\text {inv-cma }}\left(1^{k}\right)=1\right]-1 / 2\right|
$$

Definition 3 A CUS scheme is said to be invisible if for all polynomial time algorithms $\mathcal{A}$ the advantage $\operatorname{Adv}_{S, \mathcal{A}}^{i n v-c m a}$ is negligible in the security parameter $k$.

Note that the only requirement in the above definition is that $\mathcal{A} \operatorname{did}$ not submit $\left(m^{*}, \sigma^{*}\right)$ to the convert or confirm/disavow oracles. Hence, a deterministic scheme cannot satisfy the above definition since access to the signing oracle is not restricted (i.e. for a deterministic scheme, an adversary can simply submit $m^{*}$ to $\mathcal{O}_{\text {Sign }}$ and compare the received signature with $\sigma^{*}$ ). Furthermore, $\mathcal{A}$ is allowed to submit $\left(m^{*}, \sigma\right)$ where $\sigma \neq \sigma^{*}$, and $\left(m, \sigma^{*}\right)$ where $m \neq m^{*}$ to the convert and confirm/disavow oracles. While a deterministic scheme should be able to satisfy a security definition where these type of queries are allowed, some security models (e.g. [39]) do not allow the former type of query, and thereby further weaken the obtain security. These issues might be a concern in a scenario where the entropy of the signed messages is small i.e. the security of a signature on a message which the signer has previously signed might not be guaranteed. However, with the above security notion, these concerns are eliminated.

Another aspect of the above security notion which we would like to highlight is the definition of $\mathcal{S}$. We note that anyone can sample $\mathcal{S}$ and that when using this definition of $\mathcal{S}$, invisibility implies anonymity i.e. the inability for an adversary to distinguish between signatures constructed by different users (see $[16,22]$ for a formal proof of this). Some schemes (e.g. 


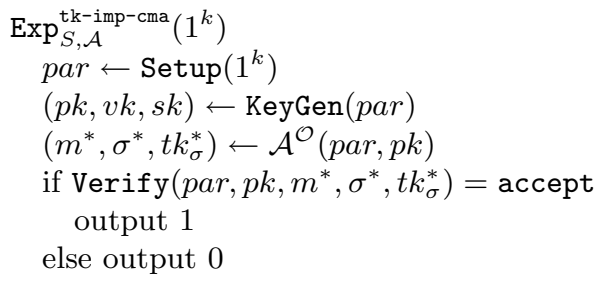

Fig. 3. Token non-impersonation experiment

$[15,23,21,20])$ use a more restricted definition limiting $\mathcal{S}$ to signatures from the signer i.e. $\mathcal{S}=\{\sigma: m \leftarrow \mathcal{M} ; \sigma \leftarrow \operatorname{Sign}(p a r, s k, m)\}$. This not only removes the guarantee of anonymity, but might also make it difficult for users other than the signer to sample $\mathcal{S}$. The latter can potentially have an impact on the non-transferability of the scheme, which we will discuss below. Regarding anonymity, we note that in the recent scheme by Huang and Wong [20], it is in fact easy to link valid signatures to a signer. This can be seen as follows: A public key in [20] is given by $p k=\left(g, X=g^{x}, Y=g^{1 / y}, u, k\right)$, where $g$ is a generator of a group $\mathbb{G}$ of order $p$ and $u \in \mathbb{G}$ is a random element, and a signature is given by $\sigma=\left(\sigma_{1}, \sigma_{2}, \sigma_{3}\right)=\left(H_{k}(m)^{1 /(x+s)}, Y^{s}, u^{s}\right)$ for a random value $s \in \mathbb{Z}_{p}$. Hence, if $\sigma$ is a valid signature, it can be checked whether $p k$ is the public key of the signer by checking whether $e\left(Y, \sigma_{3}\right)=e\left(\sigma_{2}, u\right)$ holds.

A note on non-transferability. The security notion non-transferability captures the property that a verifier who learns whether a given signature is valid or not by interacting with the signer in the confirm or disavow protocols, should not be able to prove this knowledge to a third party. More specifically, the verify should be able to "fake" any evidence of the validity of a signature obtain by interacting with the signer. When introducing convertible undeniable signatures, Boyar, Chaum, Damgård and Pedersen [6] referred to this property as undeniability and defined the property as a verifier's ability to produce fake signature/transcript pairs indistinguishable from real ones.

The above definition of invisibility guarantees that a valid signature from a signer cannot be distinguished from any other element in $\mathcal{S}$, and allows any user to sample $\mathcal{S}$. Furthermore, since the confirm and disavow protocols are required to be zero-knowledge proofs, transcript simulatability of these protocols is guaranteed. Hence, it is easy to see that a verifier can produce fake signature/transcript pairs indistinguishable from real ones, implying that nontransferability is already achieved with the above security requirements.

\subsection{Non-impersonation}

While soundness informally guarantees that a prover cannot "cheat", it does not prevent a third party from impersonating the prover. This was pointed out by Kurosawa and Heng [24], and Huang et al. [22] furthermore noticed that, for convertible schemes, this might be an issue for token generation as well.

Non-impersonation against a chosen message attack for a CUS scheme $S$ is defined via the experiment $\operatorname{Exp}_{S, \mathcal{A}}^{\text {imp-cma }}\left(1^{k}\right)$ shown in Figure 2. In the experiment, $\mathcal{A}$ has access to the oracles $\mathcal{O}=\left\{\mathcal{O}_{\text {Sign }}, \mathcal{O}_{\text {Conv }}, \mathcal{O}_{\text {Conf } / \text { Dis }}\right\}$ defined as the invisibility definition. It is required that $\mathcal{A}$ does not submit $\left(m^{*}, \sigma^{*}\right)$ to the confirm/disavow oracle. We define the advantage of $\mathcal{A}$ in the experiment as

$$
\operatorname{Adv}_{S, \mathcal{A}}^{\text {imp-cma }}=\operatorname{Pr}\left[\operatorname{Exp}_{S, \mathcal{A}}^{\text {imp-cma }}\left(1^{k}\right)=1\right]
$$


Definition 4 A CUS scheme is said to be resistant to impersonation attacks if for all polynomial time algorithms $\mathcal{A}$, the advantage $\operatorname{Adv}_{S, \mathcal{A}}^{\text {imp-cma }}$ is negligible in the security parameter $k$.

Token non-impersonation against a chosen message attack for a CUS scheme $S$ is defined via the experiment $\operatorname{Exp}_{S, \mathcal{A}}^{\mathrm{tk}-\mathrm{imp} \text {-cma }}\left(1^{k}\right)$ shown in Figure 3. $\mathcal{A}$ has access to the oracles $\mathcal{O}=$ $\left\{\mathcal{O}_{\text {Sign }}, \mathcal{O}_{\text {Conv }}, \mathcal{O}_{\text {Conf/Dis }}\right\}$ defined as in the above. It is required that $\mathcal{A}$ does not submit $\left(m^{*}, \sigma^{*}\right)$ to the conversion oracle. The advantage of $\mathcal{A}$ in the experiment is defined as

$$
\operatorname{Adv}_{S, \mathcal{A}}^{\mathrm{tk}-\mathrm{imp}-\mathrm{cma}}=\operatorname{Pr}\left[\operatorname{Exp}_{S, \mathcal{A}}^{\mathrm{tk}-\mathrm{imp}-\mathrm{cma}}\left(1^{k}\right)=1\right]
$$

Definition 5 A CUS scheme is said to be resistant to token impersonation attacks if for all polynomial time algorithms $\mathcal{A}$, the advantage $\operatorname{Adv}_{S, \mathcal{A}}^{t k-i m p-c m a}$ is negligible in the security parameter $k$.

\section{A Concrete Convertible Undeniable Signature Scheme}

In this section we present a CUS scheme provable secure in the standard model. Our scheme is based on a similar approach to the basic designated confirmer signature scheme by Schuldt et al. [36], but we employ different proof systems and provide a token generation method. Furthermore, our scheme does not require verifiers to hold public/private key pairs and avoids the key registration requirement of [36]. In the description of the scheme we use the notation $Z K P K\{w: R(w)\}$ to mean a zero-knowledge proof of knowledge of $w$ such that the relation $R(w)$ holds. We discuss the implementation details of these proofs below.

- Setup: Compute $\mathbb{P}=\left(e, \mathbb{G}_{1}, \mathbb{G}_{2}, \mathbb{G}_{T}, p, \psi\right) \leftarrow \mathcal{G}\left(1^{k}\right)$, pick $g_{2} \in \mathbb{G}_{2}$ and set $g_{1} \leftarrow \psi\left(g_{2}\right)$. Furthermore, choose a collision resistant hash function family $\mathcal{H}=\left\{H_{\mathrm{k}}:\{0,1\}^{*} \rightarrow \mathbb{Z}_{p}\right\}$ indexed by a key $\mathrm{k} \in \mathcal{K}$. Return par $=\left(\mathbb{P}, g_{1}, g_{2}, \mathcal{H}\right)$.

- KeyGen : Given par, pick $\alpha, x, y \leftarrow \mathbb{Z}_{p}, h \leftarrow \mathbb{G}_{1}$ and $w_{2} \leftarrow \mathbb{G}_{2}$, and set $w_{1} \leftarrow g_{1}^{\alpha}$, $v_{1} \leftarrow g_{2}^{x^{-1}}$ and $v_{2} \leftarrow g_{2}^{y^{-1}}$. Furthermore, pick $u_{0}, \ldots, u_{n} \leftarrow \mathbb{G}_{2}^{2}$, and define $F(m)=$ $u_{0} \prod_{i=1}^{n} u_{i}^{m_{i}}$ where $m_{i}$ is the $i$ th bit of $m$. Finally pick a hash key $\mathrm{k} \in \mathcal{K}$ and set $p k=$ $\left(\mathrm{k}, w_{1}, w_{2}, v_{1}, v_{2}, h, u_{0}, \ldots, u_{n}\right), v k=(x, y)$ and $s k=w_{2}^{\alpha}$. Return $(p k, v k, s k)$.

- Sign : Given input $($ par, $s k, m)$, where $s k=w_{2}^{\alpha}$, pick random $a, b, s \leftarrow \mathbb{Z}_{p}$, compute $t \leftarrow H_{\mathrm{k}}\left(\psi\left(v_{1}\right)^{a} \| \psi\left(v_{2}\right)^{b}|| m\right)$ and $M=g_{1}^{s} h^{t}$, and return

$$
\sigma=\left(\psi\left(v_{1}\right)^{a}, \psi\left(v_{2}\right)^{b}, \psi\left(w_{2}^{\alpha} F(M)^{a+b}\right), s\right) .
$$

- Convert : Given $(p a r, v k, m, \sigma)$ where $\sigma=\left(\sigma_{1}, \sigma_{2}, \sigma_{3}, s\right)$ and $v k=(x, y)$, check that $e\left(\sigma_{3}, g_{2}\right)=e\left(w_{1}, w_{2}\right) e\left(\sigma_{1}^{x} \sigma_{2}^{y}, F(M)\right)$ where $M=g_{1}^{s} h^{t}$ and $t=H_{\mathrm{k}}\left(\sigma_{1}\left\|\sigma_{2}\right\| m\right)$, and return $\perp$ if this is not the case. Otherwise, return the token $t k_{\sigma}=\left(\sigma_{1}^{x}, \sigma_{2}^{y}\right)$.

- Verify: Given $\left(p a r, p k, m, \sigma, t k_{\sigma}\right)$ where $p k=\left(\mathrm{k}, w_{1}, w_{2}, v_{1}, v_{2}, h, u_{0}, \ldots, u_{n}\right), \sigma^{\prime}=\left(\sigma_{1}, \sigma_{2}, \sigma_{3}, s\right)$ and $t k_{\sigma}=\left(t k_{1}, t k_{2}\right)$, return accept if

$$
\begin{aligned}
& e\left(t k_{1}, v_{1}\right)=e\left(\sigma_{1}, g_{2}\right), \quad e\left(t k_{2}, v_{2}\right)=e\left(\sigma_{2}, g_{2}\right), \quad \text { and } e\left(\sigma_{3}, g_{2}\right)=e\left(w_{1}, w_{2}\right) e\left(t k_{1} t k_{2}, F(M)\right) \\
& \text { where } M=g_{1}^{s} h^{t} \text { and } t=H_{\mathrm{k}}\left(\sigma_{1}\left\|\sigma_{2}\right\| m\right) .
\end{aligned}
$$

\footnotetext{
${ }^{2}$ We assume that the description of group elements in $\mathbb{G}_{1}$ is less than $n$ bits.
} 
- (Confirm, $\left.\mathrm{V}_{\text {con }}\right)$ : Given the common input (par, pk, $\left.m, \sigma\right)$, where $\sigma=\left(\sigma_{1}, \sigma_{2}, \sigma_{3}, s\right)$ and $p k=\left(\mathrm{k}, w_{1}, w_{2}, v_{1}, v_{2}, h, u_{0}, \ldots, u_{n}\right)$, and the additional private input $v k=(x, y)$ to the Confirm algorithm, (Confirm, $V_{\text {con }}$ ) is executed as

$$
Z K P K\left\{(x, y): v_{1}^{x}=g_{2} \wedge v_{2}^{y}=g_{2} \wedge e\left(\sigma_{1}, F(M)\right)^{x} e\left(\sigma_{2}, F(M)\right)^{y}=e\left(\sigma_{3}, g_{2}\right) / e\left(w_{1}, w_{2}\right)\right\}
$$

where $M=g_{1}^{s} h^{t}$ and $t=H_{\mathrm{k}}\left(\sigma_{1}\left\|\sigma_{2}\right\| m\right)$.

- (Disavow, $\mathrm{V}_{\text {dis }}$ ): Given same common input as in (Confirm, $V_{\text {con }}$ ) and private input $v k=$ $(x, y)$ to the Disavow algorithm, (Disavow, $\left.V_{d i s}\right)$ is executed as

$$
Z K P K\left\{(x, y): v_{1}^{x}=g_{2} \wedge v_{2}^{y}=g_{2} \wedge e\left(\sigma_{1}, F(M)\right)^{x} e\left(\sigma_{2}, F(M)\right)^{y} \neq e\left(\sigma_{3}, g_{2}\right) / e\left(w_{1}, w_{2}\right)\right\}
$$

where $M=g_{1}^{s} h^{t}$ and $t=H_{\mathrm{k}}\left(\sigma_{1}\left\|\sigma_{2}\right\| m\right)$.

Implementation of $Z K P K$. We note that it is possible to construct sigma protocols for the confirm and disavow protocols by using a combination of well known sigma protocols for proving knowledge of a discrete logarithm, equality of discrete logarithms, and inequality of discrete logarithms (see $[8,7]$ ). The zero-knowledge proofs of knowledge in the above scheme can then be obtained by using the transformation proposed by Cramer et al. [12] which converts a sigma protocol into a perfect zero-knowledge proof of knowledge. The resulting zero-knowledge proofs are efficient 4-move protocols, and no additional hardness assumptions are required in the transformation. In Appendix A, we give a more detailed description of how the protocols are implemented.

\subsection{Security}

The soundness and the zero-knowledge property of the confirm and disavow protocols is guaranteed by the transformation by Cramer et al. [12], and we refer the reader to [12] for proofs of these properties.

The token soundness of the scheme is implied by the properties of the bilinear map in combination with the proof of knowledge property of the disavow protocol (which is achieved without requiring any intractability assumptions [12]).

Theorem 6 If the disavow protocol is a zero-knowledge proof of knowledge, then the above CUS scheme has token soundness.

The proof is given in Appendix C.

The unforgeability of the above CUS scheme is based on the unforgeability of the signature scheme by Waters [38] which we recall in Appendix B.

Theorem 7 Assume that the hash function family $\mathcal{H}$ is collision resistant, the discrete logarithm problem is hard in $\mathbb{G}_{2}$, and that Waters signature scheme is (weakly) unforgeable. Then the above CUS scheme is strongly unforgeable.

We note that Waters' signature scheme is (weakly) unforgeable assuming the computational co-Diffie-Hellman problem is hard [38]. Furthermore, collision resistant hash functions can be constructed based on the discrete logarithm assumption [14], which imply that the unforgeability of the above scheme can be reduced to the computational co-Diffie-Hellman problem alone. The proof of the theorem is given in Appendix D. 
Theorem 8 Assume the above CUS scheme is strongly unforgeable and that the decision linear problem is hard in $\mathbb{G}_{2}$. Then the above CUS scheme is invisible.

The proof of the above theorem is given in Appendix E.

Lastly, the following theorems show that the above CUS scheme is resistant to impersonation and token impersonation attacks.

Theorem 9 Assume the above CUS scheme is strongly unforgeable and that the the discrete logarithm problem is hard in $\mathbb{G}_{2}$. Then the above CUS scheme is resistant to impersonation attacks.

Theorem 10 Assume the above CUS scheme is strongly unforgeable and that the computational $\psi$-Diffie-Hellman problem is hard in $\left(\mathbb{G}_{1}, \mathbb{G}_{2}\right)$. Then the above CUS scheme is resistant to token impersonation attacks.

The strategy of the proof of the first theorem is to make use of the proof of knowledge property of the used proof systems to extract the verification key which, in the simulation, will contain an unknown discrete logarithm. Otherwise, the simulation is very similar to that of Theorem 8 , and can easily be derived from this. The strategy of the proof of the second theorem is to return a signature in one of the adversary's signature queries such that a conversion will reveal the solution to a computational $\psi$-Diffie-Hellman problem ${ }^{3}$. The strong unforgeability of the scheme will ensure that the signature converted by the adversary in a token impersonation attack was constructed by the simulator, and the remaining part of the simulation is similar to that of Theorem 8. We omit the details of the proofs here.

\section{Comparison}

In Table 1 we compare the recently proposed standard model CUS schemes $[40,34,33,20]$ and our proposed scheme described above. All schemes support selective and universal conversion and are instantiated to provide approximately 80-bits of security. Note that the scheme YALS requires both a verification key and a signature of a one-time signature scheme to be included as part of a undeniable signature, which leads to a slightly larger signature size.

While the schemes PKO-4 and HW have slightly smaller signatures than our scheme, they do not provide both token soundness and anonymity. Hence, our scheme provides the smallest signature size of the CUS schemes which provably satisfies all desired security requirements. Furthermore, we note that the security of our scheme rests on weaker and more natural security assumptions compared to those of PKO-4 and HW.

Lastly we note that while delegation of verification is discussed as an extension to the scheme HW, the confirm and disavow protocols defined for the schemes YALS, PKO-3 and PKO-4 make use of the same private key material as signature generation. Delegation of verification for PKO-1 and PKO-2 is not discussed in [34], but it seems that these schemes can be extended to provide this.

\footnotetext{
${ }^{3}$ More specifically, a simulator given $\mathbb{P}$ and a $\psi$-Diffie-Hellman instance $g, g^{a}, h^{\prime} \in \mathbb{G}_{2}$ will set $g_{2} \leftarrow g^{a}$, $v_{1} \leftarrow g, u_{i} \leftarrow g^{d_{i}}$ with random $d_{i} \leftarrow \mathbb{Z}_{p}$ for $0 \leq i \leq n$, and generate the remaining elements of par and $p k$ as in an ordinary scheme. Then, for a message $m$, the signature $\sigma=\left(\sigma_{1}, \sigma_{2}, \sigma_{3}, s\right)$ constructed by picking $r, b, s \leftarrow \mathbb{Z}_{p}$, setting $\sigma_{1} \leftarrow \psi\left(h^{\prime}\right)^{r}, \sigma_{2} \leftarrow \psi\left(v_{2}\right)^{b}, t \leftarrow H_{\mathrm{k}}\left(\sigma_{1}\left\|\sigma_{2}\right\| m\right), M \leftarrow g_{1}^{s} h^{t}$ (where $h$ is from $p k$ ) and $\sigma_{3} \leftarrow \psi\left(w_{2}^{\alpha}\left(h^{\prime}\right)^{r\left(d_{0}+\sum_{i=1}^{n} d_{i} M_{i}\right)} F(M)^{b}\right)$, will have a conversion token of the form $t k_{\sigma}=\left(\psi\left(h^{\prime}\right)^{r a}, g_{1}^{b}\right)$ for a known value $r$.
} 


\begin{tabular}{|l|c|c|c|c|}
\hline Scheme & Signature Size & Token-Soundness & Anonymity & Assumptions \\
\hline \hline PKO-1 [34] & 2128 & yes & yes & sRSA + DNR \\
\hline PKO-2 [34] & 2048 & yes & yes & sRSA + DIV + DNR \\
\hline YALS [40] & 1180 & yes & yes & CDH + DLIN \\
\hline PKO-3 [33] & 680 & no & yes & $q$-SDH + DLIN \\
\hline PKO-4 [33] & 580 & no & yes & $q$-SDH + DLIN \\
\hline HW [20] & 510 & yes & no & $q$-HSDH + $q$-DHSDH \\
\hline ours & 680 & yes & yes & $\psi$-CDH + DLIN \\
\hline
\end{tabular}

Table 1. Comparison of CUS schemes. All pairing-based schemes are assumed to be instantiated with an elliptic curve group equipped with an asymmetric pairing using group elements of size 170 bits, and the RSA-based schemes are assumed to use an RSA group with a 1024 bit modulus. Furthermore, the discrete logarithm-based one-time signature scheme described in [1] and implemented in an elliptic curve group with group elements of size 160 bits, is used to instantiate the YALS [40] scheme. In the assumptions column, the abbreviations sRSA, DNR, DIV, $q$-SDH, DLIN, CDH, $q$-HSDH, $q$-DHSDH and $\psi$-CDH stands for strong RSA, decisional $N$-th residuosity, division intractability, $q$ strong Diffie-Hellman, decisional linear, computational Diffie-Hellman, $q$ hidden strong Diffie-Hellman, $q$ decisional hidden strong Diffie-Hellman and computational $\psi$-Diffie-Hellman (see the respective papers for a description of these assumptions).

\section{Conclusion}

We have given an updated definition and security model for convertible undeniable signature schemes which supports delegation of verification, and highlighted a new security property, token soundness, which is not formally captured by existing security models. We then proposed a new convertible undeniable signature scheme. Our scheme is the most efficient scheme in terms of signature size which provably fulfills all security requirements in the standard model, and is furthermore based on weak and natural security assumptions.

\section{References}

1. M. Abe, Y. Cui, H. Imai, and E. Kiltz. Efficient hybrid encryption from id-based encryption. Cryptology ePrint Archive, Report 2007/023, 2007. http://eprint.iacr.org/.

2. L. E. Aimani. Toward a generic construction of universally convertible undeniable signatures from pairingbased signatures. In INDOCRYPT, volume 5365 of LNCS, pages 145-157. Springer, 2008.

3. L. E. Aimani. Toward a generic construction of convertible undeniable signatures from pairing-based signatures. Cryptology ePrint Archive, Report 2009/362, 2009. http://eprint.iacr.org/.

4. L. E. Aimani and D. Vergnaud. Gradually convertible undeniable signatures. In ACNS, volume 4521 of LNCS, pages 478-496. Springer, 2007.

5. D. Boneh and M. K. Franklin. Identity-based encryption from the Weil pairing. In CRYPTO, volume 2139 of $L N C S$, pages 213-229. Springer, 2001.

6. J. Boyar, D. Chaum, I. Damgård, and T. P. Pedersen. Convertible undeniable signatures. In CRYPTO, volume 537 of $L N C S$, pages 189-205. Springer, 1990.

7. J. Camenisch and V. Shoup. Practical verifiable encryption and decryption of discrete logarithms. In CRYPTO, volume 2729 of LNCS, pages 126-144. Springer, 2003.

8. J. Camenisch and M. Stadler. Proof systems for general statements about discrete logarithms. Technical Report 260, Institute for Theoretical Computer Science, ETH Zurich, Mar. 1997.

9. D. Chaum. Zero-knowledge undeniable signatures. In EUROCRYPT, volume 473 of LNCS, pages 458-464. Springer, 1990.

10. D. Chaum and H. V. Antwerpen. Undeniable signatures. In CRYPTO, volume 435 of $L N C S$, pages 212-216. Springer, 1989.

11. R. Cramer, editor. Advances in Cryptology - EUROCRYPT 2005, Proceedings, volume 3494 of LNCS. Springer, 2005. 
12. R. Cramer, I. Damgård, and P. MacKenzie. Efficient zero-knowledge proofs of knowledge without intractability assumptions. In Public Key Cryptography, volume 1751 of LNCS, pages 354-372. SpringerVerlag, 2000.

13. R. Cramer, I. Damgård, and B. Schoenmakers. Proofs of partial knowledge and simplified design of witness hiding protocols. In CRYPTO, volume 839 of $L N C S$, pages 174-187. Springer, 1994.

14. I. Damgård. Collision free hash functions and public key signature schemes. In EUROCRYPT, volume 304 of $L N C S$, pages 203-216. Springer, 1987.

15. I. Damgård and T. P. Pedersen. New convertible undeniable signature schemes. In EUROCRYPT, volume 1070 of $L N C S$, pages 372-386. Springer, 1996.

16. S. D. Galbraith and W. Mao. Invisibility and anonymity of undeniable and confirmer signatures. In $C T$-RSA, volume 2612 of $L N C S$, pages 80-97. Springer, 2003.

17. R. Gennaro, H. Krawczyk, and T. Rabin. Rsa-based undeniable signatures. In CRYPTO, volume 1294 of LNCS, pages 132-149. Springer, 1997.

18. R. Gennaro, T. Rabin, and H. Krawczyk. Rsa-based undeniable signatures. J. Cryptology, 20(3):394, 2007.

19. O. Goldreich. Foundations of Cryptography. Cambridge University Press, 2001.

20. Q. Huang and D. S. Wong. New constructions of convertible undeniable signature schemes without random oracles. Cryptology ePrint Archive, Report 2009/517, 2009. http://eprint.iacr.org/.

21. X. Huang, Y. Mu, W. Susilo, and W. Wu. A generic construction for universally-convertible undeniable signatures. In $C A N S$, volume 4856 of $L N C S$, pages 15-33. Springer, 2007.

22. X. Huang, Y. Mu, W. Susilo, and W. Wu. Provably secure pairing-based convertible undeniable signature with short signature length. In Pairing, volume 4575 of LNCS, pages 367-391. Springer, 2007.

23. K. Kurosawa and J. Furukawa. Universally composable undeniable signature. In ICALP (2), volume 5126 of $L N C S$, pages 524-535. Springer, 2008.

24. K. Kurosawa and S.-H. Heng. 3-move undeniable signature scheme. In Cramer [11], pages 181-197.

25. K. Kurosawa and T. Takagi. New approach for selectively convertible undeniable signature schemes. In ASIACRYPT, volume 4284 of LNCS, pages 428-443. Springer, 2006.

26. F. Laguillaumie and D. Vergnaud. Short undeniable signatures without random oracles: The missing link. In INDOCRYPT, volume 3797 of LNCS, pages 283-296. Springer, 2005.

27. F. Laguillaumie and D. Vergnaud. Time-selective convertible undeniable signatures. In $C T$-RSA, volume 3376 of $L N C S$, pages 154-171. Springer, 2005.

28. M. Michels, H. Petersen, and P. Horster. Breaking and repairing a convertible undeniable signature scheme. In ACM Conference on Computer and Communications Security, pages 148-152, 1996.

29. M. Michels and M. Stadler. Efficient convertible undeniable signature schemes (extended abstract). In SAC'97, pages 231-244. Springer-Verlag, 1997.

30. T. Miyazaki. An improved scheme of the gennaro-krawczyk-rabin undeniable signature system based on rsa. In ICISC, volume 2015 of $L N C S$, pages 135-149. Springer, 2000.

31. J. Monnerat and S. Vaudenay. Generic homomorphic undeniable signatures. In ASIACRYPT, volume 3329 of LNCS, pages 354-371. Springer, 2004.

32. J. Monnerat and S. Vaudenay. Short 2-move undeniable signatures. In VIETCRYPT, volume 4341 of $L N C S$, pages 19-36. Springer, 2006.

33. L. T. Phong, K. Kurosawa, and W. Ogata. New dlog-based convertible undeniable signature schemes in the standard model. Cryptology ePrint Archive, Report 2009/394, 2009. http://eprint.iacr.org/.

34. L. T. Phong, K. Kurosawa, and W. Ogata. New rsa-based (selectively) convertible undeniable signature schemes. In AFRICACRYPT, volume 5580 of $L N C S$, pages 116-134. Springer, 2009.

35. C.-P. Schnorr. Efficient identification and signatures for smart cards. In CRYPTO, volume 435 of $L N C S$, pages 239-252. Springer, 1989.

36. J. C. N. Schuldt and K. Matsuura. On-line non-transferable signatures revisited. Cryptology ePrint Archive, Report 2009/406, 2009. http://eprint.iacr.org/.

37. J. C. N. Schuldt and K. Matsuura. An Efficient Convertible Undeniable Signature Scheme with Delegatable Verication. Cryptology ePrint Archive, Report 2009/454, 2009. http://eprint.iacr.org/.

38. B. Waters. Efficient identity-based encryption without random oracles. In Cramer [11], pages 114-127.

39. T. H. Yuen, M. H. Au, J. K. Liu, and W. Susilo. (convertible) undeniable signatures without random oracles. In ICICS, volume 4861 of LNCS, pages 83-97. Springer, 2007.

40. T. H. Yuen, M. H. Au, J. K. Liu, and W. Susilo. (convertible) undeniable signatures without random oracles. Cryptology ePrint Archive, Report 2007/386, 2007. http://eprint.iacr.org/. 


\section{A Implementation of Confirm and Disavow Protocols}

Before discussing the details of the implementation of the confirm and disavow protocols for the scheme presented in Section 5, we briefly recall the definition of a Sigma protocol.

A sigma protocol for a binary relation $R$ is a 3-move protocol between a prover and a verifier. Both prover and verifier receive a common input $x$, but the prover receives a witness $w$ such that $(x, w) \in R$ as an additional private input. In the first move of the protocol, the prover sends a "commitment" message $a$, in the second move, the verifier sends a random "challenge" message $c$, and in the final move, the prover sends a "response" message $z$. Given the response message, the verifier either accepts or rejects the proof. A sigma protocol is required to have two security properties:

- Special honest verifier zero-knowledge: There exists a simulation algorithm $\operatorname{Sim}_{\Sigma}$ that given input $x$ and a challenge message $c$, outputs an accepting transcript $(a, c, z) \leftarrow \operatorname{Sim}_{\Sigma}(x, c)$. We require that the simulated $(a, c, z)$ is perfectly indistinguishable from the transcript of a real interaction, conditioned on the event that the verifier chooses $c$ as his challenge message.

- Special soundness: There exists an algorithm WExt $\mathrm{W}_{\Sigma}$ that, given two accepting transcripts, $(a, c, z)$ and $\left(a, c^{\prime}, z^{\prime}\right)$, for input $x$ which have the same commitment message $a$ but different challenge messages $c \neq c^{\prime}$, can extract a witness $w$ such that $(x, w) \in R$.

Below, we will make use of sigma protocols for proving various relations among discrete logarithms, and we will use the notation

$$
\Sigma\left\{(y, z): g^{x}=h \wedge u^{y} v^{z}=e\right\}
$$

to mean a sigma protocol in which the prover receives the private input $(y, z)$ and proves to the verifier that the equations $g^{x}=h$ and $u^{y} v^{z}=e$ holds for group elements $g, h \in \mathbb{G}$ and $u, v, e \in \mathbb{G}^{\prime}$ where $\mathbb{G}$ and $\mathbb{G}^{\prime}$ might be different groups of the same order. Such sigma protocols are relatively straightforward to construct using the principle of the well-known protocol for proving knowledge of a discrete logarithm by Schnorr [35] as a building block, and we refer the reader to the analysis by Camenisch and Stadler [8] for more details.

To implement the zero-knowledge proofs of knowledge for the confirm and disavow protocols, we employ the transformation proposed by Cramer, Damgård and MacKenzie [12] which converts a sigma protocol into a perfect zero-knowledge proof of knowledge. As a tool, the transform makes use of the well known technique by Cramer, Damgård and Shoenmakers [13] for constructing a witness indistinguishable "OR" proof from sigma protocols i.e. a sigma protocol $\Sigma$ for relation $R$ and a sigma protocol $\Sigma^{\prime}$ for relation $R^{\prime}$ is combined into a sigma protocol, denoted $\Sigma \vee \Sigma^{\prime}$, which for common input $\left(x, x^{\prime}\right)$ and a witness $w$, proves that either $(x, w) \in R$ or $\left(x^{\prime}, w\right) \in R^{\prime}$. Given that $\Sigma$ and $\Sigma^{\prime}$ both have challenge space $\mathbb{Z}_{p}$, and if we, without loss of generality, assume that the prover knows a witness $w$ such that $(x, w) \in R$, $\Sigma \vee \Sigma^{\prime}$ is implemented as follows.

1. The prover honestly computes the first message $a$ of $\Sigma$, picks random $c^{\prime}$ and runs $\left(a^{\prime}, c^{\prime}, z^{\prime}\right) \leftarrow$ $\operatorname{Sim}_{\Sigma^{\prime}}\left(x^{\prime}, c^{\prime}\right)$. Then $\left(a, a^{\prime}\right)$ is sent to the verifier.

2. The verifier sends a randomly chosen $\bar{c}$ to prover.

3. The prover computes $c \leftarrow \bar{c}-c^{\prime} \bmod p$, respond honestly to $c$ with the message $z$ following $\Sigma$, and sends $\left(c, c^{\prime}, z, z^{\prime}\right)$ to the verifier who checks that $\bar{c}=c+c^{\prime} \bmod p$ and that $(a, c, z)$ and $\left(a^{\prime}, c^{\prime}, z^{\prime}\right)$ are accepting transcripts of $\Sigma$ and $\Sigma^{\prime}$. 
In the above protocol, the verifier is unable to determine which relation the prover holds a witness for. We refer the reader to [13] for a detailed analysis of the protocol.

The transformation for obtaining a zero-knowledge proof of knowledge works as follows. Let $\Sigma$ be a sigma protocol for a relation $R$ with messages $(a, c, z)$. Consider the following commitment scheme induced by $\Sigma$ and a fixed common input $x$ :

- To commit to a value $c$ in the challenge space of $\Sigma$, simulate a transcript $(a, c, z) \leftarrow$ $\operatorname{Sim}_{\Sigma}(x, c)$, and return $a$ as a commitment on $c$.

- To open a commitment $a$, reveal the corresponding $(c, z)$.

- A verifier verifies the opening by confirming that $(a, c, z)$ is an accepting transcript of $\Sigma$.

Furthermore, for this commitment scheme, assume there is a sigma protocol $\Sigma^{\prime}$ for proving knowledge of a committed value i.e. a sigma protocol for the relation $R^{\prime}$ with common input $x^{\prime}=(x, a)$ and witness $w^{\prime}=(c, z)$ (this is the only needed assumption). Then we can obtain a perfect zero-knowledge proof for $(x, w) \in R$, where $x$ is the common input and $w$ is the witness held by the prover, as follows:

1. Using the commitment scheme induced by $\Sigma$ and $x$, the verifier commits to a random value in the challenge space of $\Sigma$, and then proves knowledge of this value to the prover using $\Sigma^{\prime}$. (If the verifier does not provide an accepting proof, the prover will abort.) Let com denote the commitment sent by the verifier.

2. The prover then proves to the verifier that he knows either the witness $w$ or an opening of com using the sigma protocol $\Sigma \vee \Sigma^{\prime}$.

Although the above protocol is a six move protocol, it can easily be reduced to a four move protocol by combining the second and third moves of the sigma protocol in step 1 with the first and second moves of the sigma protocol in step 2 .

In [12], Cramer, Damgård and MacKenzie show that the protocol resulting from the above transformation is a perfect zero-knowledge proof of knowledge with knowledge error at most $2^{-t}$ assuming $\Sigma$ uses $t$-bit challenges. We refer the reader to [12] for the details.

To obtain a zero-knowledge proof for (Confirm, $\left.\mathrm{V}_{c o n}\right)$ with common input $(p a r, p k, \sigma, m)$, we construct the following sigma protocol:

$$
\Sigma_{S t d}\left\{(x, y): v_{1}^{x}=g_{2} \wedge v_{2}^{y}=g_{2} \wedge e\left(\sigma_{1}, F(M)\right)^{x} e\left(\sigma_{2}, F(M)\right)^{y}=e\left(\sigma_{3}, g_{2}\right) / e\left(w_{1}, w_{2}\right)\right\}
$$

where $M=g_{1}^{s} h^{t}$ and $t=H_{\mathrm{k}}\left(\sigma_{1}\left\|\sigma_{2}\right\| m\right)$. A commitment to a value $c \in \mathbb{Z}_{p}$ constructed using $\Sigma_{S t d}$ and $(p a r, p k, \sigma, m)$ will be of the form $\left(a_{1}, a_{2}, a_{3}\right)=\left(v_{1}^{z_{1}} g_{2}^{-c}, v_{2}^{z_{2}} g_{2}^{-c}, e_{1}^{z_{1}} e_{2}^{z_{2}} e_{3}^{-c}\right)$ where $e_{1}=e\left(\sigma_{1}, F(M)\right), e_{2}=e\left(\sigma_{2}, F(M)\right), e_{3}=e\left(\sigma_{3}, g_{2}\right) / e\left(w_{1}, w_{2}\right)$, and $z_{1}, z_{2} \leftarrow \mathbb{Z}_{p}$ are random values. The corresponding opening is $\left(c, z_{1}, z_{2}\right)$, and a verifier accept the opening if $\left(a_{1}, a_{2}, a_{3}, c, z_{1}, z_{2}\right)$ is a valid transcript of $\Sigma_{S t d}$. The following sigma protocol proves knowledge of an opening.

$$
\Sigma_{S t d}^{\prime}\left\{\left(c, z_{1}, z_{2}\right): v_{1}^{z_{1}} g_{2}^{-c}=a_{1} \wedge v_{2}^{z_{2}} g_{2}^{-c}=a_{2} \wedge e_{1}^{z_{1}} e_{2}^{z_{2}} e_{3}^{-c}=a_{3}\right\}
$$

Hence, by applying the above transformation to $\Sigma_{S t d}$ and $\Sigma_{S t d}^{\prime}$, a zero-knowledge proof for (Confirm, $\mathrm{V}_{c o n}$ ) is obtained.

To obtain a zero-knowledge proof for (Disavow, $\left.V_{d i s}\right)$ with common input (par, $\left.p k, m, \sigma\right)$, we apply the same strategy as above. Firstly, we construct a sigma protocol $\Sigma\left\{(x, y): v_{1}^{x}=\right.$ $\left.g_{2} \wedge v_{2}^{y}=g_{2} \wedge e_{1}^{x} e_{2}^{y} \neq e_{3}\right\}$ by adapting the technique used in the proof of inequality of discrete 
logarithms by Camenish and Shoup [7]. More specifically, a prover first chooses $r \leftarrow \mathbb{Z}_{p}$, computes $C \leftarrow\left(e_{1}^{x} e_{2}^{y} / e_{3}\right)^{r}$, and then interacts with the verifier in the protocol

$$
\bar{\Sigma}_{S t d}\left\{(\alpha, \beta, r): v_{1}^{\alpha} g_{2}^{-r}=1 \wedge v_{2}^{\beta} g_{2}^{-r}=1 \wedge e_{1}^{\alpha} e_{2}^{\beta} e_{3}^{-r}=C\right\}
$$

where $\alpha=x r$ and $\beta=y r$. A verifier will only accept a proof if $C \neq 1$. A commitment to a value $c \in \mathbb{Z}_{p}$ constructed using $\bar{\Sigma}_{S t d}$ and $(p a r, p k, \sigma, m)$ is of the form $\left(a_{1}, a_{2}, a_{3}, C\right)=$ $\left(v_{1}^{z_{\alpha}} g_{2}^{-z_{r}}, v_{2}^{z_{\beta}} g_{2}^{-z_{r}}, e_{1}^{z_{\alpha}} e_{2}^{z_{\beta}} e_{3}^{-z_{r}} C^{-c}, C\right)$ where $z_{\alpha}, z_{\beta}, z_{r} \leftarrow \mathbb{Z}_{p}$ and $C \leftarrow \mathbb{G}_{T}$ are random values. To prove knowledge of the opening $\left(c, z_{\alpha}, z_{\beta}, z_{r}\right)$, the following sigma protocol can be used

$$
\bar{\Sigma}_{S t d}^{\prime}\left\{\left(c, z_{\alpha}, z_{\beta}, z_{r}\right): v_{1}^{z_{\alpha}} g_{2}^{-z_{r}}=a_{1} \wedge v_{2}^{z_{\beta}} g_{2}^{-z_{r}}=a_{2} \wedge e_{1}^{z_{\alpha}} e_{2}^{z_{\beta}} e_{3}^{-z_{r}} C^{-c}=a_{3}\right\}
$$

Hence, by applying the above transformation to $\bar{\Sigma}_{S t d}$ and $\bar{\Sigma}_{S t d}^{\prime}$ we obtain a zero-knowledge proof for (Disavow, $\mathrm{V}_{\text {dis }}$ ).

\section{B Waters' Signature Scheme}

Below we recall the signature scheme by Waters [38]. Note that we make use of an asymmetric bilinear map $e: \mathbb{G}_{1} \times \mathbb{G}_{2} \rightarrow \mathbb{G}_{T}$ whereas the original scheme in [38] was defined using a symmetric bilinear map $e: \mathbb{G}_{1} \times \mathbb{G}_{1} \rightarrow \mathbb{G}_{T}$.

- Setup: Compute $\mathbb{P}=\left(e, \mathbb{G}_{1}, \mathbb{G}_{2}, \mathbb{G}_{T}, p, \psi\right) \leftarrow \mathcal{G}\left(1^{k}\right)$, pick $g_{2} \in \mathbb{G}_{2}$ and set $g_{1} \leftarrow \psi\left(g_{2}\right)$. Return par $=\left(\mathbb{P}, g_{1}, g_{2}\right)$.

- KeyGen : Given par, pick $\alpha \leftarrow \mathbb{Z}_{p}$ and $w_{2} \leftarrow \mathbb{G}_{2}$, and set $w_{1} \leftarrow g_{1}^{\alpha}$. Furthermore, pick $u_{0}, \ldots, u_{n} \leftarrow \mathbb{G}_{2}$, and define $F(m)=u_{0} \prod_{i=1}^{n} u_{i}^{m_{i}}$ where $m_{i}$ is the $i$ th bit of $m$. Finally set the public key to $p k=\left(w_{1}, w_{2}, u_{0}, \ldots, u_{n}\right)$ and the private key to $s k=w_{2}^{\alpha}$. Return $(p k, s k)$.

- Sign : Given input $\left(\right.$ par, sk,m), where $s k=w_{2}^{\alpha}$ pick $r \leftarrow \mathbb{Z}_{p}$, compute $\sigma_{1} \leftarrow g_{1}^{r}$ and $\sigma_{2} \leftarrow \psi\left(w_{2}^{\alpha} F(m)^{r}\right)$, and return the signature $\sigma=\left(\sigma_{1}, \sigma_{2}\right)$.

- Verify : Given par, a public key $p k=\left(w_{1}, w_{2}, u_{0}, \ldots, u_{n}\right)$, a message $m$ and a signature $\sigma=\left(\sigma_{1}, \sigma_{2}\right)$, return accept if $e\left(\sigma_{2}, g_{2}\right)=e\left(w_{1}, w_{2}\right) e\left(\sigma_{1}, F(m)\right)$.

It follows from the proof of security given in [38], that the above signature scheme is unforgeable against a chosen message attack assuming the computational co-Diffie-Hellman problem is hard in $\left(\mathbb{G}_{1}, \mathbb{G}_{2}\right)$.

\section{Proof of Theorem 6}

Proof. Firstly, we show that if a signature passes the token verification, it must be a valid signature. Consider a public key $p k=\left(\mathrm{k}, w_{1}, w_{2}, v_{1}, v_{2}, h, u_{0}, \ldots, u_{n}\right)$, a purported signature $\sigma=\left(\sigma_{1}, \sigma_{2}, \sigma_{3}, s\right)$ on a message $m$, and a token $t k_{\sigma}=\left(t k_{1}, t k_{2}\right)$. There must exist unique values $a, b \in \mathbb{Z}_{p}$ such that $\sigma_{1}=\psi\left(v_{1}\right)^{a}$ and $\sigma_{2}=\psi\left(v_{2}\right)^{b}$. Then, if the verification equations $e\left(t k_{1}, v_{1}\right)=e\left(\sigma_{1}, g_{2}\right)$ and $e\left(t k_{2}, v_{2}\right)=e\left(\sigma_{2}, g_{2}\right)$ hold, we must have that $t k_{1}=g_{1}^{a}$ and $t k_{2}=g_{1}^{b}$. Hence, we have $t k_{1} t k_{2}=g_{1}^{a+b}$. Furthermore, if $e\left(\sigma_{3}, g_{2}\right)=e\left(w_{1}, w_{2}\right) e\left(g_{1}^{a+b}, F(M)\right)$, where $M=g_{1}^{s} h^{t}$ and $t=H_{\mathrm{k}}\left(\sigma_{1}\left\|\sigma_{2}\right\| m\right)$, also holds, we have that $\sigma_{3}=\psi\left(w_{2}^{\alpha} F(M)^{a+b}\right)$, where $\alpha=\log _{g_{1}} w_{1}$. Hence, if the output of Verify $\left(p a r, p k, m, \sigma, t k_{\sigma}\right)$ is accept, $\sigma$ must be a valid signature on $m$ under the public key $p k$. 
Now assume that the adversary completes the disavow protocol for $(p k, m, \sigma)$ with nonnegligible probability. By the proof of knowledge property of the protocol, there exists a knowledge extractor which is able to extract a witness $(\alpha, \beta, r)$ with non-negligible probability such that $v_{1}^{\alpha}=g_{2}^{r}, v_{2}^{\beta}=g_{2}^{r}$ and $e_{1}^{\alpha} e_{2}^{\beta} / e_{3}^{r}=C$ for some $C \neq 1$, where $e_{1}=e\left(\sigma_{1}, F(M)\right)$, $e_{2}=e\left(\sigma_{2}, F(M)\right)$, and $e_{3}=e\left(\sigma_{3}, g_{2}\right) / e\left(w_{1}, w_{2}\right)$. Letting $x=\log _{v_{1}} g_{2}$ and $y=\log _{v_{2}} g_{2}$, this implies that $\alpha=x r$ and $\beta=y r$. Hence, we must have that $\left(e_{1}^{x} e_{2}^{y} / e_{3}\right)^{r} \neq 1$ which implies that $e_{1}^{x} e_{2}^{y} \neq e_{3}$. However, this contradicts that $\sigma$ is a valid signature on $m$ under $p k$.

\section{Proof of Theorem 7}

Proof. Assume that a successful adversary $\mathcal{A}$ that breaks the unforgeability of the CUS scheme exists. Let $\left(m^{*}, \sigma^{*}\right)$ denote the forgery output by $\mathcal{A}$ where $\sigma^{*}=\left(\sigma_{1}^{*}, \sigma_{2}^{*}, \sigma_{3}^{*}, s^{*}\right)$, and let $\left(m_{i}, \sigma_{i}\right)$ denote the $i$ th sign query and response where $\sigma_{i}=\left(\sigma_{i, 1}, \sigma_{i, 2}, \sigma_{i, 3}, s_{i}\right)$. Furthermore, we let $M^{*}=g_{1}^{s^{*}} h^{t^{*}}, t^{*}=H_{\mathrm{k}}\left(\sigma_{1}^{*}\left\|\sigma_{2}^{*}\right\| m^{*}\right), M_{i}=g_{1}^{s_{i}} h^{t_{i}}$, and $t_{i}=H\left(\sigma_{i, 1}\left\|\sigma_{i, 2}\right\| m_{i}\right)$, where $h$ is part of the public key $p k$. Finally, let $q$ be the total number of sign queries made the adversary. We then define three different types of forgeries:

1. A forgery where $M^{*}=M_{i}$ and $t^{*}=t_{i}$ for some $i \in\{1, \ldots, q\}$.

2. A forgery where $M^{*}=M_{i}$ and $t^{*} \neq t_{i}$ for some $i \in\{1, \ldots, q\}$.

3. A forgery where $M^{*} \neq M_{i}$ for all $i \in\{1, \ldots, q\}$.

If $\mathcal{A}$ is successful, he must produce a forgery of one of the above types. For each type, we define algorithms $\mathcal{B}_{1}, \mathcal{B}_{2}$ and $\mathcal{B}_{3}$ that breaks the collision resistance of $H_{\mathrm{k}}$, solves the discrete logarithm problem in $\mathbb{G}_{2}$, and breaks the weak unforgeability of the Waters signature scheme, respectively.

Type $1 . \mathcal{B}_{1}$ 's goal for this category forgery is, given a description of a hash function family $\mathcal{H}$ and a random hash key $\mathrm{k} \in \mathcal{K}$, to produce messages $x_{1} \neq x_{2}$ such that $H_{\mathrm{k}}\left(x_{1}\right)=H_{\mathrm{k}}\left(x_{2}\right)$. We construct $\mathcal{B}_{1}$ as follows: Firstly, $\mathcal{B}_{1}$ runs $\mathbb{P}=\left(e, \mathbb{G}_{1}, \mathbb{G}_{2}, \mathbb{G}_{T}, p, \psi\right) \leftarrow \mathcal{G}\left(1^{k}\right)$, picks $g_{2} \leftarrow \mathbb{G}_{2}$ and sets $g_{1} \leftarrow \psi\left(g_{2}\right)$ and par $\leftarrow\left(\mathbb{P}, g_{1}, g_{2}, \mathcal{H}\right)$. Then $\mathcal{B}_{1}$ runs $(p k, v k, s k) \leftarrow \operatorname{KeyGen}($ par $)$ but uses the received hash key $\mathrm{k}$ in $p k$ instead of picking a random key. Then $\mathcal{B}_{1}$ runs $\mathcal{A}$ with input $(p a r, p k, v k)$.

While running, $\mathcal{A}$ can ask sign queries $m_{i}$ which $\mathcal{B}_{1}$ responds to by returning $\sigma_{i} \leftarrow$ $\operatorname{Sign}\left(p a r, s k, m_{i}\right)$. Eventually, $\mathcal{A}$ outputs a forgery $\left(m^{*}, \sigma^{*}\right)$ where $\sigma^{*}$ is of the form $\left(\sigma_{1}^{*}, \sigma_{2}^{*}, \sigma_{3}^{*}, s^{*}\right)$. $\mathcal{B}_{1}$ outputs messages $x_{1}=\sigma_{1}^{*}\left\|\sigma_{2}^{*}\right\| m^{*}$ and $x_{2}=\sigma_{i, 1}\left\|\sigma_{i, 2}\right\| m_{i}$ where $i$ is the index for which $M^{*}=M_{i}$ and $t^{*}=t_{i}$.

$\mathcal{B}_{1}$ succeeds if $x_{1} \neq x_{2}$. Assume towards a contradiction that $x_{1}=x_{2}$ i.e. $\sigma_{1}^{*}\left\|\sigma_{2}^{*}\right\| m^{*}=$ $\sigma_{i, 1}\left\|\sigma_{i, 2}\right\| m_{k}$. Since $\mathcal{A}$ 's forgery is a type 1 forgery, we have that $M_{i}=g^{s_{i}} h^{t_{i}}=g^{s^{*}} h^{t^{*}}=M^{*}$ and $t_{i}=t^{*}$. This implies that $s_{i}=s^{*}$. Furthermore, if $\sigma^{*}$ is a valid signature, it must be possible to write $\sigma_{3}^{*}$ as $\psi\left(w_{2}^{\alpha} F\left(M^{*}\right)^{a+b}\right)$ where $a=\log _{v_{1}} \sigma_{1}^{*}$ and $b=\log _{v_{2}} \sigma_{2}^{*}$. However, since $\sigma_{1}^{*}=\sigma_{i, 1}, \sigma_{2}^{*}=\sigma_{i, 2}$ and $M^{*}=M_{i}$, we must have $\sigma_{i, 3}=\psi\left(w_{2}^{\alpha} F\left(M_{i}\right)^{a+b}\right)=\sigma_{3}^{*}$. Hence, we have $\left(\sigma_{1}^{*}, \sigma_{2}^{*}, \sigma_{3}^{*}, s^{*}\right)=\left(\sigma_{i, 1}, \sigma_{i, 2}, \sigma_{i, 3}, s_{i}\right)$ which contradicts $\mathcal{A}$ outputting a valid forgery.

Type 2. $\mathcal{B}_{2}$ 's goal in this category is, given $\mathbb{P}=\left(e, \mathbb{G}_{1}, \mathbb{G}_{2}, \mathbb{G}_{T}, p, \psi\right)$ and elements $g_{2}, h_{2} \in \mathbb{G}_{2}$, to compute $x=\log _{g_{2}} h_{2}$. We construct $\mathcal{B}_{2}$ as follows: Firstly, $\mathcal{B}_{2}$ picks a hash function family $\mathcal{H}$, sets $g_{1} \leftarrow \psi\left(g_{2}\right)$ and $p a r \leftarrow\left(\mathbb{P}, g_{1}, g_{2}, \mathcal{H}\right)$, and runs $(p k, v k, s k) \leftarrow \operatorname{KeyGen}($ par $)$ but uses the element $h \leftarrow \psi\left(h_{2}\right)$ in $p k$ instead of picking a random element in $\mathbb{G}_{1}$. Then $\mathcal{B}_{2}$ runs $\mathcal{A}$ with input (par, pk,vk). 
While running, $\mathcal{A}$ can ask sign queries which $\mathcal{B}_{2}$ responds to by returning $\sigma_{i} \leftarrow \operatorname{Sign}\left(\right.$ par, sk, $\left.m_{i}\right)$. Eventually, $\mathcal{A}$ outputs a forgery $\left(m^{*}, \sigma^{*}\right)$. Since $\mathcal{A}$ 's forgery is assumed to be of type 2 , there must be a $i$ such that $M_{i}=g_{1}^{s_{i}} h^{t_{i}}=g_{1}^{s^{*}} h^{t^{*}}=M^{*}$ but $t_{i} \neq t^{*}$. Hence, $\mathcal{B}_{2}$ can compute $x=\log _{g_{1}} h=\log _{g_{2}} h_{2}=\left(s_{i}-s^{*}\right) /\left(t^{*}-t_{i}\right)$.

Type 3. $\mathcal{B}_{3}$ 's goal in this category is to produce a forgery of the signature scheme by Waters. $\mathcal{B}_{3}$ interacts with a (weak) unforgeability challenger $\mathcal{C}$ which provides $\mathcal{B}_{3}$ with a signing oracle. Initially, $\mathcal{B}_{3}$ is given parameters $p a r=\left(\mathbb{P}, g_{1}, g_{2}, \mathcal{H}\right)$ and a public key $p k^{\prime}=\left(w_{1}, w_{2}, u_{0}, \ldots, u_{n}\right)$. Firstly, $\mathcal{B}_{3}$ creates a public key $p k$ by picking a hash key $\mathrm{k} \in \mathcal{K}$, picking values $c, x, y \leftarrow \mathbb{Z}_{p}$, setting $h \leftarrow g_{1}^{c}, v_{1} \leftarrow g_{2}^{x^{-1}}$ and $v_{2} \leftarrow g_{2}^{y^{-1}}$, and finally setting $p k=\left(\mathrm{k}, w_{1}, w_{2}, v_{1}, v_{2}, h, u_{0}, \ldots, u_{n}\right)$. Then $\mathcal{B}_{3}$ sets $v k \leftarrow(x, y)$ and runs $\mathcal{A}$ with input (par, pk,vk).

While running, $\mathcal{A}$ can ask sign queries, which $\mathcal{B}_{3}$ responds to as follows:

- Sign queries: Given a message $m, \mathcal{B}_{3}$ picks random $z \leftarrow \mathbb{Z}_{p}$, sets $M \leftarrow g_{1}^{z}$ and queries $M$ to its signing oracle to obtain a Waters signature $\sigma^{\prime}=\left(g_{1}^{r}, \psi\left(w_{2}^{\alpha} F(M)^{r}\right)\right)$ on $M$. Then $\mathcal{B}_{3}$ picks random $r^{\prime} \leftarrow \mathbb{Z}_{p}$, and computes $\sigma_{1} \leftarrow\left(g_{1}^{r} g_{1}^{r^{\prime}}\right)^{x^{-1}}=\psi\left(v_{1}\right)^{r+r^{\prime}}$ and $\sigma_{2} \leftarrow$ $\left(g_{1}^{r} / g_{1}^{r^{\prime}}\right)^{y^{-1}}=\psi\left(v_{2}\right)^{r-r^{\prime}}$. Note that since both $r$ and $r^{\prime}$ will be uniformly distributed in $\mathbb{Z}_{p}$, so will $r+r^{\prime}$ and $r-r^{\prime}$ (like the values $a$ and $b$ in an ordinary signature). Lastly, $\mathcal{B}_{3}$ computes $s \leftarrow z-c H_{\mathrm{k}}\left(\sigma_{1}\left\|\sigma_{2}\right\| m\right)$, sets $\sigma=\left(\sigma_{1}, \sigma_{2}, \psi\left(w_{2}^{\alpha} F(M)^{r}\right), s\right)$, and returns $\sigma$ to $\mathcal{A}$. Note that $s$ is uniformly distributed in $\mathbb{Z}_{p}$ and that $M=g^{s} h^{H_{\mathrm{k}}\left(\sigma_{1}\left\|\sigma_{2}\right\| m\right)}$. Hence, $\sigma$ is a valid signature on $m$, and is distributed as in the original unforgeability game.

Eventually, $\mathcal{A}$ outputs a forgery $\left(m^{*}, \sigma^{*}\right)$ where $\sigma^{*}=\left(\sigma_{1}^{*}, \sigma_{2}^{*}, \sigma_{3}^{*}, s^{*}\right)$. $\mathcal{B}_{3}$ then computes $\sigma_{1}^{\prime} \leftarrow$ $\left(\sigma_{1}^{*}\right)^{x}\left(\sigma_{2}^{*}\right)^{y}$ and outputs the message $M^{*}$ and $\sigma^{\prime} \leftarrow\left(\sigma_{1}^{\prime}, \sigma_{3}^{*}\right)$. Note that if $\mathcal{A}^{\prime}$ 's forgery is valid, it follows that $e\left(\sigma_{3}^{*}, g_{2}\right)=e\left(w_{1}, w_{2}\right) e\left(\sigma_{1}^{\prime}, F\left(M^{*}\right)\right)$, and since $\mathcal{A}$ 's forgery is assumed to be a type 3 forgery, we have that $M^{*} \neq M_{i}$ for all $i$. Hence, the validity of $\mathcal{B}_{3}$ 's forgery follows directly from the validity of $\mathcal{A}$ 's forgery, and $\mathcal{B}_{3}$ successfully attacks the weak unforgeability of the Waters signature scheme whenever $\mathcal{A}$ successfully attacks the CUS scheme.

\section{E Proof of Theorem 8}

Proof. We assume that an adversary $\mathcal{A}$ breaking the invisibility of the CUS scheme exists. Let forge be the event that $\mathcal{A}$ submits a convert or confirm/disavow query $(m, \sigma)$ where $\sigma$ is a valid signature on $m$ which was not obtained through a sign query $m$. In a successful attack by $\mathcal{A}$, either forge or $\neg$ forge will occur. In the following we will construct algorithms $\mathcal{B}_{1}$ and $\mathcal{B}_{2}$ which will break the strong unforgeability of the scheme if forge occurs, and the decisional linear assumption if $\neg$ forge occurs and $\mathcal{A}$ is successful, respectively.

First assume that the event forge happens. $\mathcal{B}_{1}$ runs an unforgeability experiment, receives the input (par, pk,vk), and forwards $(p a r, p k)$ as input to $\mathcal{A}$. While running, $\mathcal{A}$ can ask sign, convert and confirm/disavow queries. $\mathcal{B}_{1}$ responds to these queries as follows. If $\mathcal{A}$ makes a sign query, $\mathcal{B}_{1}$ forwards this query to his own signing oracle, and returns the obtained signature to $\mathcal{A}$. If $\mathcal{A}$ makes a convert or confirm/disavow query $(m, \sigma), \mathcal{B}_{1}$ first checks if $\sigma$ was returned as a response to a sign query on $m$. If this is not the case, $\mathcal{B}_{1}$ checks if $(m, \sigma)$ is valid (using $v k$ ), and if so, returns $(m, \sigma)$ as a forgery and halts. Otherwise, $\mathcal{B}_{1}$ either returns $t k_{\sigma} \leftarrow$ Convert $(p a r, v k, m, \sigma)$ or $\perp$, or interacts with $\mathcal{A}$ running Confirm or Disavow, depending on the query type and the validity of $(m, \sigma)$.

At some point, $\mathcal{A}$ outputs a challenge message $m^{*}$. As in the invisibility experiment, $\mathcal{B}_{1}$ flips a random coin $b \leftarrow\{0,1\}$ and returns a random $\sigma^{*} \leftarrow \mathcal{S}$ if $b=0$. Otherwise, $\mathcal{B}_{1}$ returns $\sigma^{*}$ 
obtained by submitting $m^{*}$ to his own signing oracle. After receiving $\sigma^{*}, \mathcal{A}$ can ask additional sign, convert and confirm/disavow queries which $\mathcal{B}_{1}$ answers as above. If forge happens, it is clear that $\mathcal{B}_{1}$ succeeds in winning in the unforgeability experiment.

Now assume that forge does not happen. $\mathcal{B}_{2}$ will attempt to solve the decisional linear assumption i.e. $\mathcal{B}_{2}$ receives $\mathbb{P}=\left(e, \mathbb{G}_{1}, \mathbb{G}_{2}, \mathbb{G}_{T}, p, \psi\right)$ and elements $u, v, u^{x}, v^{y}, h, h^{z} \in \mathbb{G}_{2}$. $\mathcal{B}_{2}$ 's goal is to decide if $z=x+y$. Firstly, $\mathcal{B}_{2}$ picks a hash family $\mathcal{H}=\left\{H_{\mathrm{k}}:\{0,1\}^{*} \rightarrow \mathbb{Z}_{p}\right\}$ and an element $g_{2} \leftarrow \mathbb{G}_{2}$, and sets $g_{1} \leftarrow \psi\left(g_{2}\right)$ and par $\leftarrow\left(\mathbb{P}, g_{1}, g_{2}, \mathcal{H}\right)$. $\mathcal{B}_{2}$ then generates a public key by choosing $\alpha \leftarrow \mathbb{Z}_{p}$ and $w_{2} \leftarrow \mathbb{G}_{2}$, and setting $w_{1} \leftarrow g_{1}^{\alpha}, v_{1} \leftarrow u, v_{2} \leftarrow v$ and $h_{1} \leftarrow \psi(h)$. Furthermore, $\mathcal{B}_{2}$ picks a hash key $\mathrm{k} \in \mathcal{K}$ and $d_{0}, \ldots, d_{n} \leftarrow \mathbb{Z}_{p}$, and sets $u_{i} \leftarrow h^{d_{i}}$ for $1 \leq i \leq n$, $p k \leftarrow\left(\mathrm{k}, w_{1}, w_{2}, v_{1}, v_{2}, h_{1}, u_{0}, \ldots, u_{n}\right)$ and $s k=w_{2}^{\alpha}$. Lastly, $\mathcal{B}_{2}$ runs $\mathcal{A}$ with input $(p a r, p k)$.

While running, $\mathcal{A}$ can ask sign, convert and confirm/disavow queries which are answered as follows.

- Sign: Given a message $m, \mathcal{B}_{2}$ returns $\sigma=\left(\psi\left(v_{1}\right)^{a}, \psi\left(v_{2}\right)^{b}, \psi\left(w_{2}^{\alpha} F(M)^{a+b}\right), s\right) \leftarrow \operatorname{Sign}($ par, sk, $m)$ but remember the random choices $a, b \leftarrow \mathbb{Z}_{p}$ and stores $(m, \sigma, a, b)$.

- Convert: Given $(m, \sigma), \mathcal{B}_{2}$ checks if $\sigma$ was returned as a response to a sign query $m$. If this is not the case, $\mathcal{B}_{2}$ returns $\perp$. Otherwise, $\mathcal{B}_{2}$ recalls the random choices $a, b$ used to construct $\sigma$, and returns $t k_{\sigma}=\left(g_{1}^{a}, g_{1}^{b}\right)$.

- Confirm/Disavow: Given $(m, \sigma), \mathcal{B}_{2}$ simulates the confirm protocol if $\sigma$ was returned as a response to a sign query on $m$, but simulates the disavow protocol otherwise.

To simulate the confirm protocol, $\mathcal{B}_{2}$ interacts with $\mathcal{A}$ as follows. Upon receiving a commitment com (constructed using $\Sigma_{S t d}$ ) and the first message $a$ of $\Sigma_{S t d}^{\prime}$ from $\mathcal{A}, \mathcal{B}_{2}$ choose a random challenge $c \leftarrow \mathbb{Z}_{p}$, computes the first message $a^{\prime}$ of $\Sigma_{S t d} \vee \Sigma_{S t d}^{\prime}$ as if an opening of com is known (note that the computation of the first message $a^{\prime}$ does not require knowledge of an opening to com), and returns $\left(a^{\prime}, c\right)$ to $\mathcal{A}$. When $\mathcal{A}$ responds with the last message $z$ of $\Sigma_{S t d}^{\prime}$ and a challenge $c^{\prime}$ for $\mathcal{B}_{2}, \mathcal{B}_{2}$ checks if $(a, c, z)$ is an accepting transcript of $\Sigma_{S t d}$. If not, $\mathcal{B}_{2}$ returns $\perp$ to $\mathcal{A}$. Otherwise, $\mathcal{B}_{2}$ rewinds $\mathcal{A}$ and provides $\mathcal{A}$ with a new challenge $\bar{c} \leftarrow \mathbb{Z}_{p}$ for the $\Sigma_{\text {Std }}^{\prime}$ protocol. Hence, if $\mathcal{A}$ responds with a message $\bar{z}$ such that $(a, \bar{c}, \bar{z})$ is a valid transcript for $\Sigma_{S t d}^{\prime}, \mathcal{B}_{2}$ can extract an opening of com and complete the protocol $\Sigma_{S t d} \vee \Sigma_{S t d}^{\prime}$ honestly for any challenge $c^{\prime}$ sent by $\mathcal{A}$ (i.e. $\mathcal{B}_{2}$ learns a witness for $\Sigma_{S t d}^{\prime}$ and can therefore honestly run $\left.\Sigma_{S t d} \vee \Sigma_{S t d}^{\prime}\right)$. If $(a, \bar{c}, \bar{z})$ is an invalid transcript, $\mathcal{B}_{2}$ returns $\perp$ to $\mathcal{A}$.

The simulation of the disavow protocol is similar to the above, except the protocols $\bar{\Sigma}_{S t d}^{\prime}$ and $\bar{\Sigma}_{S t d} \vee \bar{\Sigma}_{S t d}^{\prime}$ are used.

At some stage, $\mathcal{A}$ outputs a challenge message $m^{*}$. $\mathcal{B}_{2}$ constructs a challenge signature by picking $s^{*} \leftarrow \mathbb{Z}_{p}$ and computing $t^{*} \leftarrow H_{\mathrm{k}}\left(\psi\left(u^{x}\right)\left\|\psi\left(v^{y}\right)\right\| m^{*}\right), M^{*} \leftarrow g_{1}^{s^{*}} h_{1}^{t^{*}}$ and $\sigma^{*} \leftarrow$ $\left(\psi\left(u^{x}\right), \psi\left(v^{y}\right), \psi\left(w_{2}^{\alpha}\left(h^{z}\right)^{d_{0}+\sum_{i=1}^{n} d_{i} M_{i}^{*}}\right), s^{*}\right)$, where $\left(u^{x}, v^{y}, h^{z}\right)$ are the elements received in the decisional linear problem and $h_{1}$ is from $p k$. Note that if $z$ is random, then $\sigma^{*}$ will be a random element in $\mathbb{G}_{1}^{3} \times \mathbb{Z}_{p}$, whereas if $z=x+y, \sigma^{*}$ will be a valid signature on $m^{*}$ since $\left(h^{z}\right)^{d_{0}+\sum_{i=1}^{n} d_{i} M_{i}^{*}}=\left(h^{d_{0}+\sum_{i=1}^{n} d_{i} M_{i}^{*}}\right)^{x+y}=\left(u_{0} \prod_{i=1}^{n} u_{i}^{M_{i}^{*}}\right)^{x+y}=F\left(M^{*}\right)^{x+y}$.

$\mathcal{B}_{2}$ returns $\sigma^{*}$ to $\mathcal{A}$ who can then ask additional sign, convert and confirm/disavow queries, but is not allowed to query $\sigma^{*}$ to the convert or confirm/disavow oracle. $\mathcal{B}_{2}$ answers these queries as above. Eventually, $\mathcal{A}$ outputs a bit $b$ which $\mathcal{B}_{2}$ forwards as his own solution to the decisional linear problem.

$\mathcal{B}_{2}$ 's simulation of the invisibility experiment for $\mathcal{A}$ is perfect if forge does not happen, and it is clear that $\mathcal{B}_{2}$ will solve the decisional linear problem if $\mathcal{A}$ breaks the invisibility of the scheme. 